Advances in Geosciences, 2, 285-292, 2005

SRef-ID: 1680-7359/adgeo/2005-2-285

European Geosciences Union

(c) 2005 Author(s). This work is licensed

under a Creative Commons License.

\title{
Orographic influences in rainfall downscaling
}

\author{
M. G. Badas, R. Deidda, and E. Piga \\ Dipartimento di Ingegneria del Territorio, Università di Cagliari, Piazza d'Armi, 09123 Cagliari, Italy
}

Received: 14 December 2004 - Revised: 10 February 2005 - Accepted: 10 February 2005 - Published: 15 August 2005

\begin{abstract}
The problem of rainfall downscaling in a mountainous region is discussed, and a simple methodology aimed at introducing spatial heterogeneity induced by orography in downscaling models is proposed. This procedure was calibrated and applied to rainfall data retrieved by the high temporal resolution rain gage network of the Sardinian Hydrological Survey.
\end{abstract}

\section{Introduction}

The development of effective procedures for precipitation downscaling has a prominent role in the forecast of hydrogeological risk for small watersheds. Actually an efficient application of rainfall-runoff models requires the knowledge of precipitation over space and time scales which are smaller than the ones resolved by meteorological models. Thus the rainfall volume (or equivalently the mean rainfall intensity), which has been forecasted by meteorological models over large space and time scales, needs to be disaggregated up to the smallest scales of hydrologic models. In Fig. 1 the domain of a downscaling process is illustrated: $L$ and $T$ are the space and time scales resolved by the meteorological models, $\lambda_{0}$ and $\tau_{0}$ are the smallest scales of the hydrologic resolution, which are compatible with the watershed extension.

Statisical downscaling procedures allow describing the small scale uncertainty in the localization of rainfall peaks in space and time. A source of uncertainty is indeed related to the gap of scales between meteorological and hydrological models. This is certainly true when the meteorological model resolution is larger than the required hydrological scales, and it may still remain true when limited area models (LAMs) are nested into general circulation models (GCMs) in order to increase the resolved resolutions. Indeed, the coarse resolution of the observation network used to build the meteorological analyses, on which the initial conditions for partial differen-

Correspondence to: R. Deidda

(rdeidda@unica.it) tial equations solved by GCMs are based, cannot allow to correctly localize and quantify rainfall peaks in high resolution space-time domains, where LAMs are usually nested. Thus also in these cases statistical downscaling procedures can be applied as a tool to account for small scales uncertainties.

Also other sources of model uncertainties may affect a forecasting operational chain. The ensemble prediction system (EPS) that is currently running at the ECMWF (European Center for Medium range Weather Forecasting) allows accounting for meteorological model uncertainty due to the coarseness of the observation network and to the chaoticity of equations used in meteorological models (Buizza et al., 1999). As the last element of a forecasting chain, hydrological models are also affected by uncertainties that are mainly due to our limited knowledge of catchment subsurface properties (Beven, 2001). In this paper we focus the problem of rainfall downscaling with special regard to mountain regions, while for a discussion of the different sources of model uncertainties the reader is referred to Ferraris et al. (2002), since it goes beyond the scope of this work.

An important class of statistical models useful for rainfall downscaling is represented by multifractal models, which are able to reproduce rainfall fields observed variability at different space and time scales. Statistical properties of rainfall fields in space and time are usually characterized by means of multifractal theory applying self-similar or self-affine transformations. When self-similarity holds scale invariance can be investigated introducing a constant parameter $U$, having the dimension of a velocity, which allows statistical properties observed at spatial scales $\lambda$ to be equivalent to the properties at time scales $\tau=\lambda / U$. For the general case of self-affine fields Lovejoy and Schertzer (1985) introduced the G.S.I. (Generalized Scale Invariance) formalism, where the equivalence between statistical properties observed in space and time can be pursued by means of a velocity parameter $U \propto \lambda^{H}$ function of $\lambda$ scale, where $H$ is defined as the anisotropy scaling exponent. The condition $H=0$ corresponds to the self-similar hypothesis. 

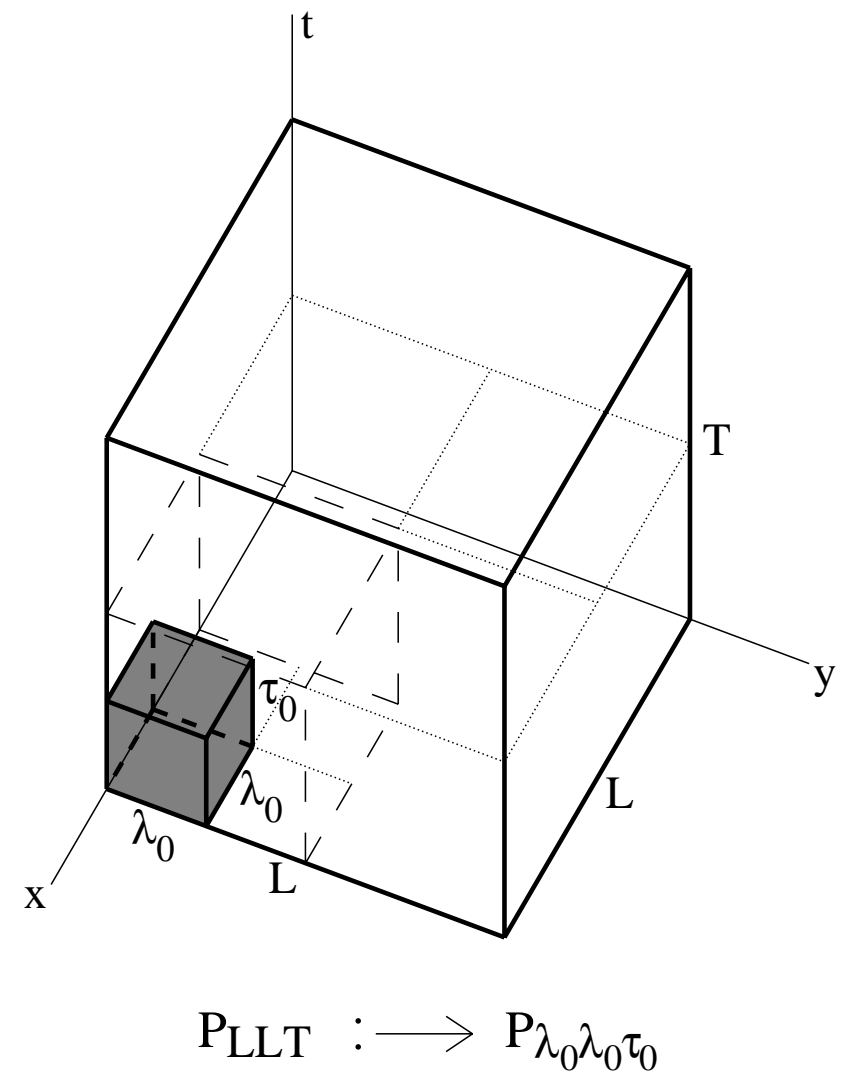

Fig. 1. A schematization of a downscaling problem: a rainfall measure is given over a region $L \times L \times T$ (e.g., the grid resolution of a NWP model), we want to determine the probability distribution of rainfall intensity over any smaller region $\lambda_{0} \times \lambda_{0} \times \tau_{0}$ (Deidda, 2000).

Some recent studies performed on radar rainfall datasets retrieved during the two international campaigns GATE (GARP, Global Atmospheric Reasearch Program, Atlantic Tropical Experiment) and TOGA-COARE (Tropical Ocean Global Atmosphere Coupled Ocean-Atmosphere Response Experiment) gave evidence of a scale invariance behavior with self-similar transformations (Deidda, 2000; Deidda et al., 2004).

Both GATE and TOGA-COARE precipitation fields were retrieved over the ocean without any physical obstacle which could bias the spatial distribution of rainfall probability in the analyzed domain. On the contrary, when precipitation fields need to be generated in presence of orographic obstacles and barriers, it is convenient to analyze the effects of these constraints on rainfall fields and on local probability rainfall distributions. Actually mean and extreme precipitation values are known to vary with the terrain elevation. More generally local probability distribution of rainfall may be influenced by many morphological factors besides the altitude: slope and shape of orographic relieves, as well as their exposition with respect to the direction of the perturbation. Nevertheless it may be rather difficult to deduct the influence of each factor from the analysis of point rainfall data retrieved by rain gages.
The opportunity of introducing a heterogeneous component when modeling synthetic rainfall fields over land was also investigated by Harris et al. (1996), Jothityangkoon et al. (2000), Purdy et al. (2001), Pathirana and Herath (2002). Specifically Jothityangkoon et al. (2000) and Pathirana and Herath (2002) analyzed a $400 \mathrm{~km} \times 400 \mathrm{~km}$ area in southwestern Australia and a $128 \mathrm{~km} \times 128 \mathrm{~km}$ region centered in the Japanese archipelago respectively. In order to reproduce observed spatial heterogeneity they multiplied a homogeneous spatial random cascade by a deterministic factor depending on the spatial location without linking it to local features. Analyzing a transect along the Southern Alps of New Zeland, Harris et al. (1996) and Purdy et al. (2001) found a dependence of scaling parameters on orography and rain features. More recently Deidda et al. (2005) investigated the presence of rainfall spatial heterogeneity induced by orography on rainfall fields retrieved over Brazil by radars (TRMM-LBA, Tropical Rainfall Measurement Mission - Large Scale Biosphere Atmosphere Experiment). Nevertheless these analyses did not highlight the presence of any spatial heterogeneity, probably because the limited differences in altitude of the examined domain were not sufficient to force significant upward air movements.

In the present paper the analysis of the orographic influence on precipitation observed by the high temporal resolution rain gage network of the Sardinian Hydrological Survey is discussed. The examined data were collected in the period from 1986 to 1996 by 235 pluviometric stations (Fig. 2), which recorded on solid memory the instants when $0.2 \mathrm{~mm}$ of rainfall were cumulated. With the aim to perform a scale invariance analysis and to validate the downscaling procedure, rain gage data were averaged on the cells of a regular grid. The grid spatial step $\lambda_{0}=13 \mathrm{~km}$ was obtained dividing the island width $L=104 \mathrm{~km}$ by an integer power of two, with at least one rain gage in most of the cells. The comparison of self-correlation functions in space and time allowed to determine the velocity parameter $U=17.33 \mathrm{~km} / \mathrm{h}$ needed for self-similar transformations. Therefore the smallest time resolution is $\tau_{0}=\lambda_{0} / U=45 \mathrm{~min}$, while the examined event duration is $T=L / U=6 \mathrm{~h}$. Figure 2 shows the domains and the grid used for both the space-time scale invariance analysis and the downscaling process.

The present work is organised as follows. In Sect. 2 we present a methodology to introduce local constraints in the rainfall disaggregation by means of a modulating function. This function is determined in Sect. 3 on the basis of some preliminary analyses based on both rain gage observations and average rainfall on cells displayed in Fig. 2. In Sect. 4 the scale-invariance analysis performed on space-time domains is described, in Sect. 5 the results obtained for numerical simulations are discussed and finally in Sect. 6 the conclusions of the work are drawn. 


\section{Introduction of local heterogeneity in synthetic rain- fall fields}

We propose a simple methodology allowing to investigate spatial heterogeneity in rainfall fields and to introduce corresponding constraints in downscaling models. Let us consider the domain $L \times L \times T$ of Fig. 1 whose mean rainfall intensity $I$ for each precipitation event may be computed as follows:

$I=\frac{1}{L L T} \int_{0}^{L} \int_{0}^{L} \int_{0}^{T} i(x, y, t) d x d y d t$

where $i(x, y, t)$ is rainfall intensity at location $(x, y)$ and time $t$. The ratio between mean rainfall intensity of each cell $(x, y)$ and the mean rainfall $I$ in this whole domain represents a local modulating function expressed by:

$\xi(x, y)=\frac{\frac{1}{T} \int_{0}^{T} i(x, y, t) d t}{I}$

The function $\xi(x, y)$ shows high variability due to the effect of orography and to the specific feature of each precipitation event. Averaging $\xi(x, y)$ on many events and analyzing the sample mean $\bar{\xi}(x, y)$ filters the sample variability due to rain storm features and helps to assess the existence of orographic constraints in observed fields.

If rainfall is spatially homogeneous the expected value of the $\bar{\xi}(x, y)$ function should be equal to one for any location $(x, y)$. If the analysis highlights a dependence on localization, this represents a territorial heterogeneity which has to be reproduced also in the synthetic fields.

Following a similar approach to the one adopted by Jothityangkoon et al. (2000) and Pathirana and Herath (2002), this kind of spatial heterogeneity can be reproduced multiplying homogeneous random cascade $i_{0}(x, y, t)$ by the modulating function $\bar{\xi}(x, y)$ :

$i(x, y, t)=\bar{\xi}(x, y) i_{0}(x, y, t)$

\section{Estimate of the modulating function}

The research of a modulating function has been performed on raw rain gage data and on data gridded on the cells of the grid described in the introduction. Both the analyses were made on intense precipitation events with a duration $T$ equal to $6 \mathrm{~h}$.

\subsection{Analysis on rain gage data}

The analysis on rain gage data was performed on independent events selected on a regional scale by computing for each event the regional mean intensity $I_{p}$ on a time interval of duration $T=6 \mathrm{~h}$ :

$I_{p}\left(t^{\prime}\right)=\frac{1}{N_{p}} \sum_{k=1}^{N_{p}} \frac{1}{T} \int_{t^{\prime}}^{t^{\prime}+T} i_{k}(t) d t$

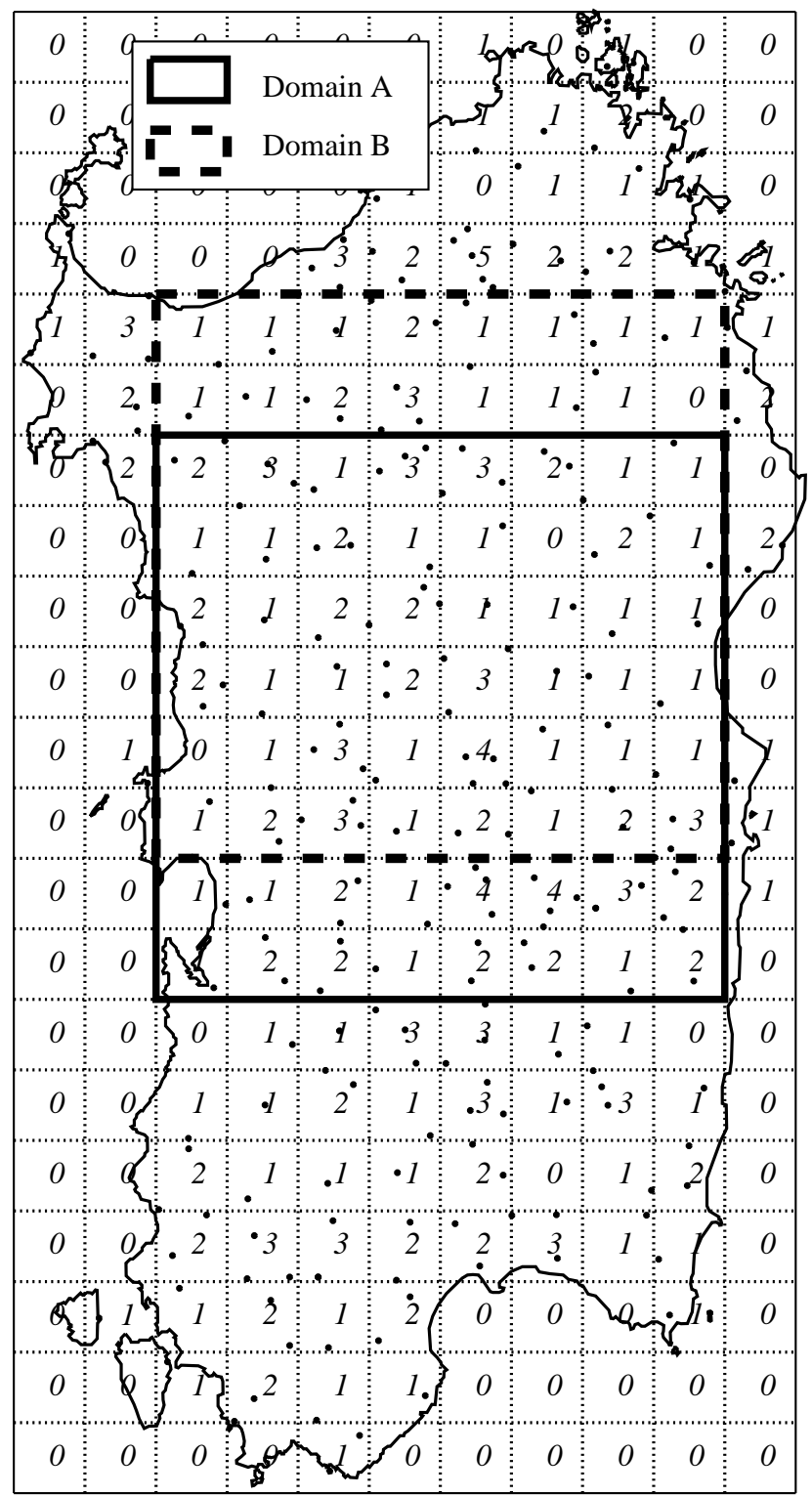

Fig. 2. Points indicating the location of the rain gages and regular grid with $13 \mathrm{~km}$ resolution used for spatial homogeneity investigation, the number of rain gages per cell is reported inside the cell. Space-time sequences used in Sects. 4 and 5 were extracted from the $\mathrm{A}$ and $\mathrm{B}$ domains.

where $N_{p}$ is the number of active recording rain gages during the precipitation event beginning at time $t^{\prime}$ and $i_{k}(t)$ is rainfall intensity retrieved by the $k$-th rain gage.

The most intense 794 independent events were selected, with average intensity $I_{p}$ ranging from $0.2 \mathrm{~mm} / \mathrm{h}$ and $4.5 \mathrm{~mm} / \mathrm{h}$. For rain gage $k$ the function $\xi(k)=\left(\frac{1}{T} \int_{t^{\prime}}^{t^{\prime}+T} i_{k}(t) d t\right) / I_{p}$ of each event and its average value on all the events $\bar{\xi}(k)$ were computed. The analysis of the results showed a spatial variability of $\bar{\xi}(k)$ function which can be partially explained considering the rain gage altitude $z(k)$. As illustrated in Fig. 3 the comparison between modulating functions $\bar{\xi}(k)$ and altitude $z(k)$ shows 


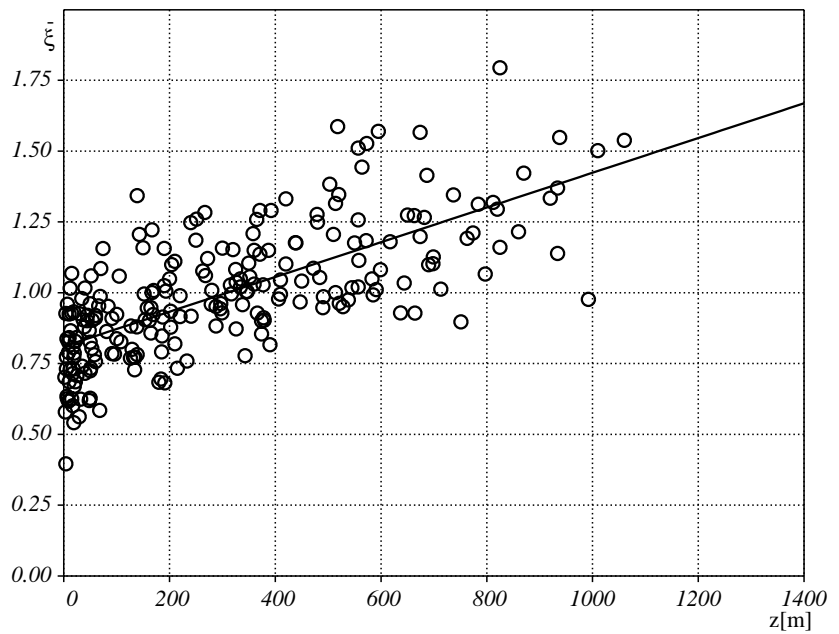

Fig. 3. Values of the modulating function $\bar{\xi}(k)$ versus rain gage altitude $z(k)$ and corresponding regression line.

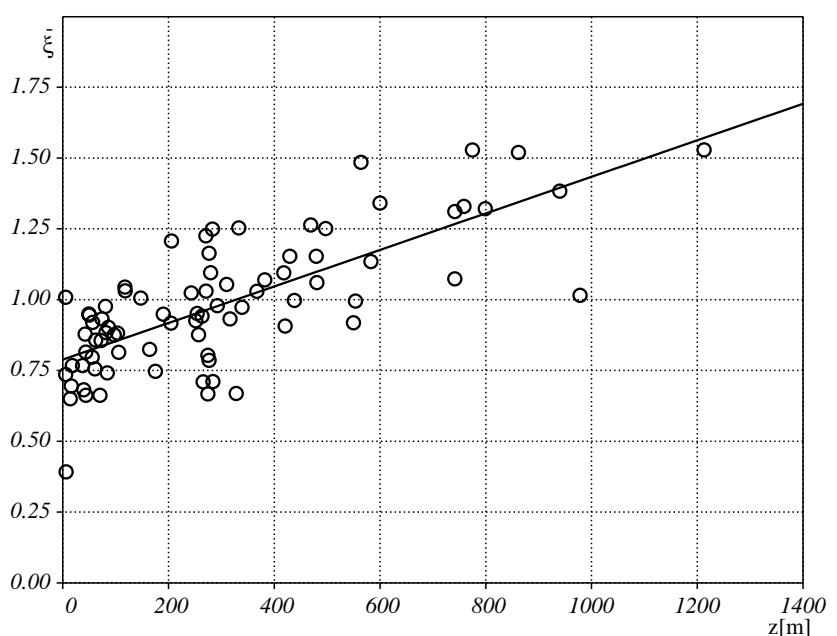

Fig. 4. Modulating function values $\bar{\xi}(x, y)$ versus mean grid cell altitude $z(x, y)$ and corresponding regression line.

a behavior which can be interpreted linearly, despite of a noticeable dispersion of the representative points. The regression line slope $\alpha$ computed on sample points and showed in Fig. 3 is equal to $0.61 / 1000 \mathrm{~m}^{-1}$.

\subsection{Analysis on a regular grid}

With the aim to obtain a feasible relationship for the analysis and the simulation of space-time rainfall events, the $\xi$ function was also examined for rainfall data averaged on the regular grid described in the introduction and displayed in Fig. 2. The regional mean intensity $I_{g}$ on $T=6 \mathrm{~h}$ and on the whole spatial grid was on this case computed as:

$I_{g}\left(t^{\prime}\right)=\frac{1}{N_{g}} \sum_{k=1}^{N_{g}} \frac{1}{T} \int_{t^{\prime}}^{t^{\prime}+T} i_{k}(t) d t$

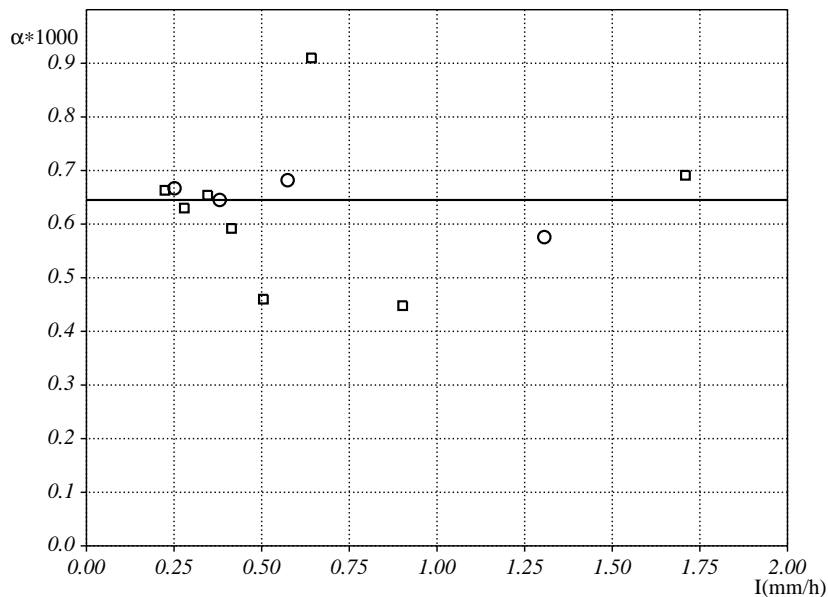

Fig. 5. Slopes $\alpha$ obtained dividing the selected events into four groups (circles) and eight groups (squares) versus mean group rain intensity. Solid line represents mean $\alpha$ value computed on all the events.

where $N_{g}$ is the number of grid cells with at least one rain gage working during the event, $t^{\prime}$ is the beginning of the event, while $i_{k}(t)$ is the average rainfall intensity of rain gages within the $k$-th grid cell.

The most intense 806 events were selected with mean intensity $I_{g}$ ranging from $0.2 \mathrm{~mm} / \mathrm{h}$ and $4.5 \mathrm{~mm} / \mathrm{h}$. Similarly to the case of raw rain gage data, for each grid cell $(x, y)$ the mean value $\bar{\xi}(x, y)$ on all the events was computed. The Fig. 4 shows the behavior of the modulating function $\bar{\xi}(x, y)$ with the average altitude $z(x, y)$ of the cell: the regression line slope $\alpha=0.65 / 1000 \mathrm{~m}^{-1}$ shows a similar value to the one obtained for single rain gage data.

With the aim to verify the stability of $\alpha$ estimates, the dataset containing 806 sequences has been divided according to intensity into four and then eight groups having the same number of elements. Mean values $\bar{\xi}(x, y)$ and slopes $\alpha$ of the regression lines (in the $\xi-z$ plane) have been computed for each group. The $\alpha$ values are plotted in Fig. 5 versus the mean rainfall intensity of the group. As these values do not show any trend with intensity, $\alpha$ has been thus considered constant and equal to its mean value $0.65 / 1000 \mathrm{~m}^{-1}$, which was estimated on the 806 events.

In order to represent the behavior of the modulating function $\bar{\xi}(x, y)$ with the average cell altitude $z(x, y)$ the following equation was assumed:

$\bar{\xi}(x, y)=\alpha z(x, y)+b$

where the intercept $b=1-\alpha<z>(x, y)$ is computed using the relationships (1) and (2), while $<\cdot>_{(x, y)}$ is the spatial average on the selected domain. In such a way it is possible to define the functional relationship between $\bar{\xi}$ and the altitude for different spatial domains. 


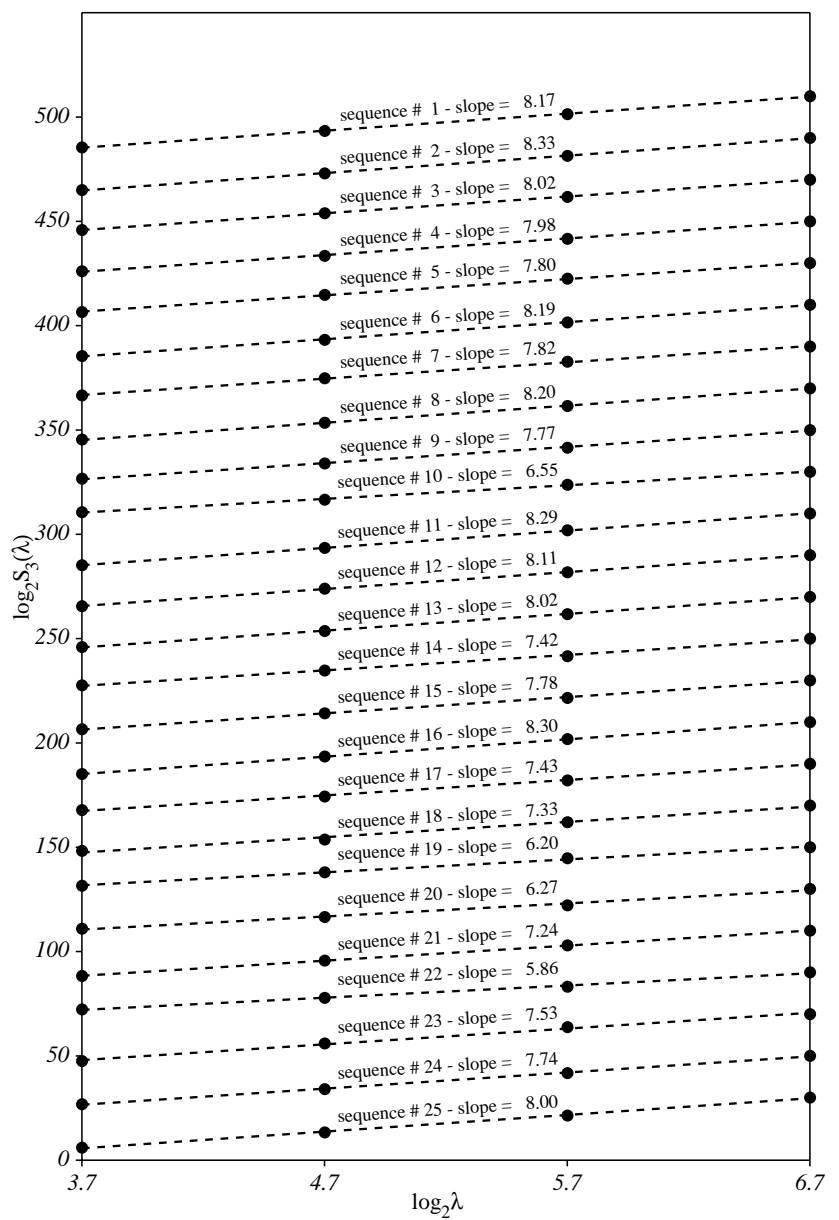

Fig. 6. Partition functions of the third order $S_{3}(\lambda)$ computed on spatial scales ranging from $\lambda_{0}=13 \mathrm{~km}$ to $L=104 \mathrm{~km}$ (and corresponding time scales from $\tau_{0}=45 \mathrm{~min}$ a $T=6 \mathrm{~h}$ ) for the 25 most intense sequences. For each sequence the multifractal exponent $\zeta(3)$, slope of the regression line, is indicated.

\section{Scale invariant properties on rainfall events extracted on a regular grid}

Due to the lack of rain gages in some cells or to their discontinuous retrieving in the examined period, some of the cell data were missing and it was necessary to fill them in order to perform the scale invariance analysis. In these cells the lacking data were estimated averaging rainfall intensities on the adjacent eight grid cells in the same time interval $\tau_{0}=45 \mathrm{~min}$.

The 138 most intense sequences defined over $L=104 \mathrm{~km}$ and $T=6 \mathrm{~h}$, with a resolution $\lambda_{0}=13 \mathrm{~km}$ and $\tau_{0}=45 \mathrm{~min}$, were selected over the A and B domains (Fig. 2) depending on where the highest intensity occurred. The mean rain intensities $I$ resulted in the range $0.2-3.5 \mathrm{~mm} / \mathrm{h}$. For the following analyses, selected sequences were made spatially homogeneous dividing rainfall intensity of each cell $(x, y)$ by the corresponding modulating function value $\bar{\xi}(x, y)$ defined by the Eq. (6) for the respective domain.

The research of scale invariant properties was thus performed using self-similar transformations and computing for
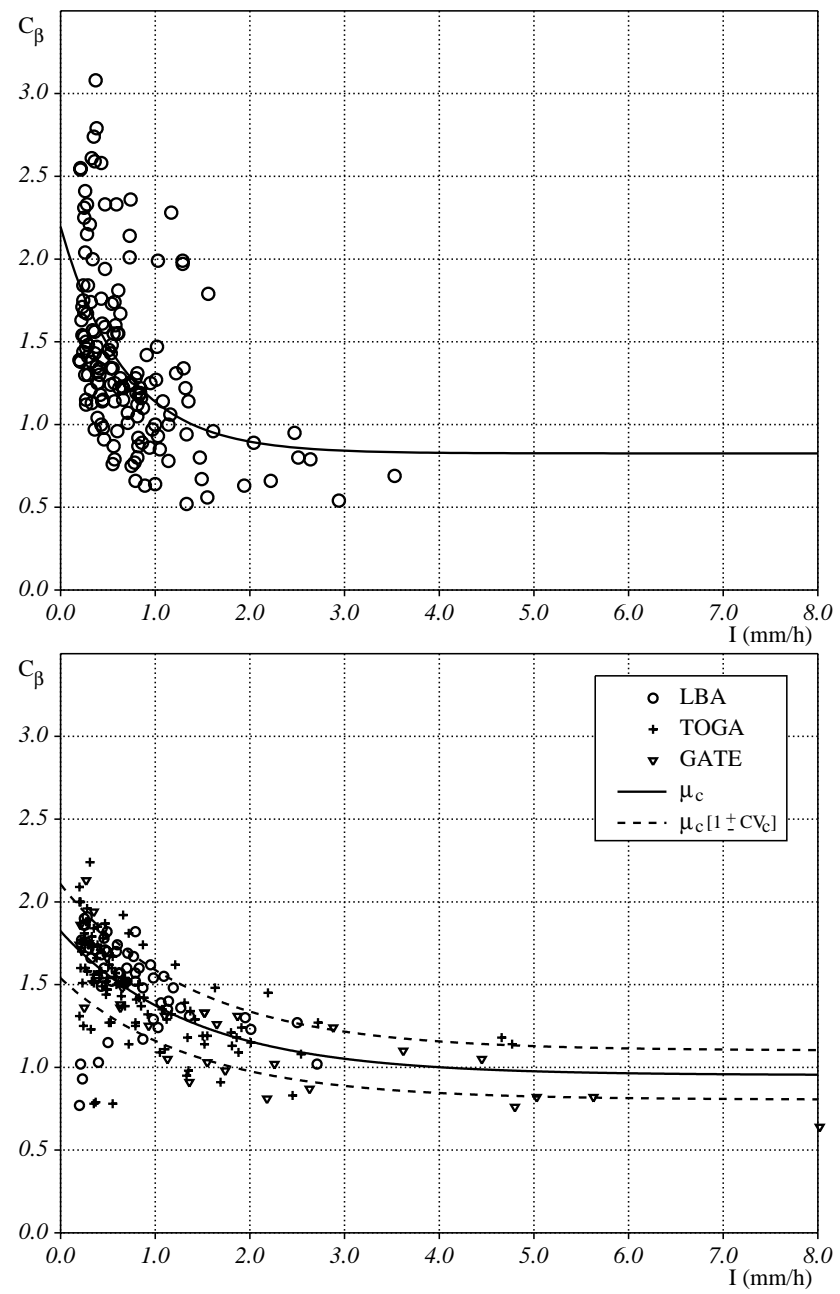

Fig. 7. Sample estimates of the $c$ parameter computed on the 138 selected sequences plotted versus mean rainfall intensity $I$ and corresponding regression line (top). Sample $c$ estimates for the sequences extracted from TRMM-LBA, TOGA and GATE datasets, corresponding regression lines $\mu(I)$ and confidence limits (bottom).

each sequence the partition functions $S_{q}(\lambda)$ on space scales $\lambda$ from $\lambda_{0}=13 \mathrm{~km}$ to $L=104 \mathrm{~km}$ and corresponding time scales $\tau$ from $\tau_{0}=\lambda_{0} / U=45 \mathrm{~min}$ to $T=L / U=6 \mathrm{~h}$ :

$S_{q}(\lambda)=\frac{1}{N(\lambda)^{2} N(\tau)} \sum_{i=1}^{N(\lambda)} \sum_{j=1}^{N(\lambda)} \sum_{k=1}^{N(\tau)} \mu_{i, j, k}(\lambda)^{q}$

where $N(\lambda)^{2} N(\tau)$ is the number of cells in the $\lambda$ partition, $\mu_{i, j, k}(\lambda)=\int_{x_{i}}^{x_{i}+\lambda} d x \int_{y_{j}}^{y_{j}+\lambda} d y \int_{t_{k}}^{t_{k}+\lambda / U} d t i(x, y, t)$, is the rainfall volume of the single cell located in $x_{i}, y_{j}, t_{k}$. The precipitation field is scale invariant if there is at least one range of scales $\lambda$ where relationships of the following type exist:

$S_{q}(\lambda) \sim \lambda^{\zeta(q)}$

The field is multifractal if $\zeta(q)$ exponents turn out to be a no-linear function of the moment $q$. The partition functions 


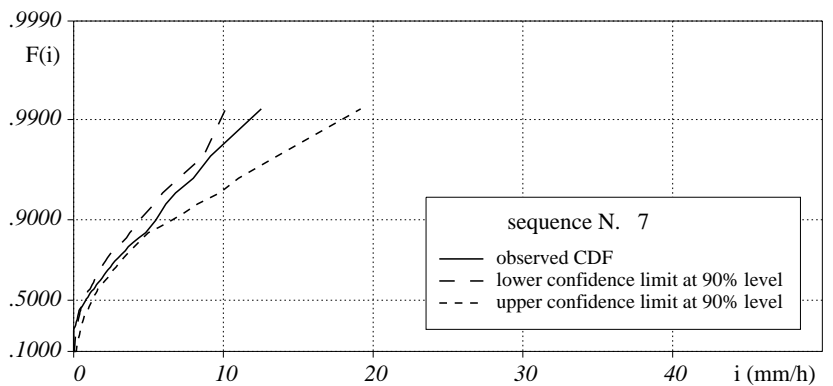

Fig. 8. Cumulative distribution function of rainfall rain rate at higher resolution is compared to the $90 \%$ confidence limits estimated from synthetic fields for one of the analyzed sequences.

$S_{q}(\lambda)$, computed for moments $q$ from 1 to 6 , showed a good correspondence to the scaling law (8) for all the space and time scales considered. As an example in Fig. 6 the third order partition functions of the most intense 25 sequences are showed: sample points are well interpreted by scaling laws (8) which correspond to linear trends in the log-log plane. The regression line slopes, given in Fig. 6, provide estimates of the $\zeta(q)$ multifractal exponents which are useful in the calibration of multifractal models, where $\zeta(q)$ expected values are generally expressed as a function of the generator parameters and of $q$.

In the present work the behavior of the multifractal exponents $\zeta(q)$ was interpreted by the STRAIN cascade model (Deidda, 2000) which is based on a log-Poisson generator $\eta=\beta^{y}$, where $y$ is a log-Poisson variable with mean $c$. Thus the expected value of the multifractal exponents results to be a function of the two model parameters $c, \beta$, other than the moment $q$ :

$\zeta(q)=d q-c \frac{q(1-\beta)-\left(1-\beta^{q}\right)}{\ln 2}$

where $d=3$ is the dimension of the analyzed fields. As already found in the GATE and TOGA-COARE datasets, $\beta$ estimates do not show a great variability among the different sequences, therefore $\beta$ was considered as constant and equal to $e^{-1}$. The $c$ parameter estimates, bounded to the constant $\beta$ value, showed (despite a high variability) a decreasing trend with increasing mean intensities. This behavior, displayed in Fig. 7 (top), was interpreted with the following equation:

$c(I)=a \exp (-\gamma I)+c_{\infty}$

where $a=1.3679, \gamma=1.4792, c_{\infty}=0.8257$.

The form of the Eq. (10) is the same of the one used to interpret the $c$ behavior for GATE, TOGA-COARE, and TRMM-LBA sequences (Deidda, 2000; Deidda et al., 2004, 2005). Estimates of the $c$ parameter obtained for these radar campaigns are displayed in Fig. 7 (bottom) together with the regression line $\mu(I)$ and the interval $c(I)=\mu(I)[1 \pm C V]$ which was determined by means of the variation coefficient $C V$. The comparison between Figs. 7 (top) and 7 (bottom) highlights that $c$ estimates obtained for sequences extracted
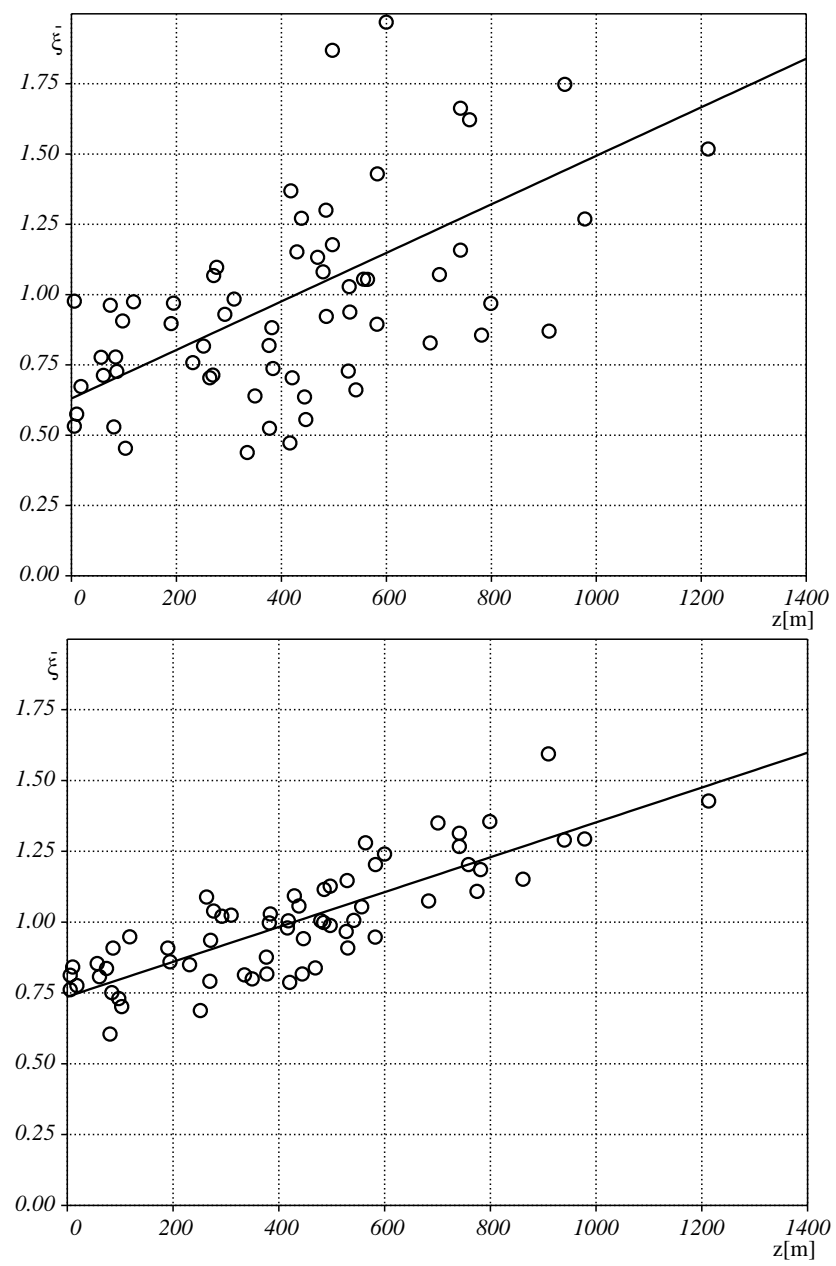

Fig. 9. Averages of the modulating function $\bar{\xi}$ plotted versus mean cell altitude $z$ for the events selected on the A domain. The plots refer to the observed sequences (top) and to one set of syntethic generations (bottom).

from rain gages in Sardinia are moved away from the ones obtained from radar data. This outcome might depend on the difference in analyzed data, concerning their type and discretization, as well as on the specific features of the examined region. Despite the analyzed sequences had similar scale invariant properties, a unique equation does not seem able to interpret $c$ parameter behavior. Thus in the following sections we use Eq. (10) calibrated on Sardinian stations (top of Fig. 7).

\section{Simulation of synthetic fields}

In order to verify the effectiveness of the proposed downscaling procedure, a set of 100 synthetic fields was generated for each of the 138 sequences using the STRAIN model with parameters $\beta=e^{-1}$ and $c$ obtained from Eq. (10) for the same rainfall intensity $I$ of the corresponding observed field.

The selected observed sequences were made spatially homogeneous by dividing them by $\bar{\xi}(x, y)$ function. A first 
comparison was performed among these sequences and synthetic homogeneous ones. Cumulative distribution functions (CDF) of local rainfall intensity at the smallest scales $(13 \mathrm{~km}$ and $45 \mathrm{~min}$ ) computed for the observed sequences were compared to the $90 \%$ confidence intervals at obtained from the corresponding set of synthetic sequences.

Sample CDF are within this interval for most of the analyzed fields, meaning a correct reproduction of the observed behavior. As an example the CDF corresponding to one of the selected sequence and its $90 \%$ confidence intervals are displayed in Fig. 8.

Spatial heterogeneity was later introduced in homogeneous synthetic fields by multiplying rainfall intensity in each cell $(x, y)$ by the same modulating function $\bar{\xi}(x, y)$ given by Eq. (6). The dependence of the cell mean intensity values on the altitude was verified on observed and simulated sequences over the $\mathrm{A}$ and $\mathrm{B}$ domains.

In Figs. 9 (top) and 10 (top) sample behavior of the average parameter $\bar{\xi}$ as a function of the altitude is showed for the observed events on the A and B domains. A visual examination of these figures confirms a dependence of $\bar{\xi}$ on the altitude, despite a notable dispersion of the data. The regression line slope is $\alpha=0.86 / 1000 \mathrm{~m}^{-1}$ for the A domain and $\alpha=0.40 / 1000 \mathrm{~m}^{-1}$ for the B domain, therefore the slope is, in both cases, different from the one obtained for the entire region in Sect. 3 analysing more than 800 events.

It is important to notice that selected sequences are 70 on the A domain and 68 on the B domain and that the analyses performed on a regional scale showed a reasonable variability in the $\alpha$ estimate with the number of events used for $\xi$ averaging. As showed in Fig. 5, the dispersion of $\alpha$ estimates made on sets of 100 events (square symbols) is comparable to the one obtained for the data belonging to the A and B domains. It is therefore difficult to understand whether differences in the two domains, which are superimposed for three quarters, are due to a different local behavior or to the high variability in $\alpha$ estimates computed on a small group of events.

\section{Conclusions}

The orographic influence in rainfall fields has been investigated on data retrieved by the high temporal resolution rain gage network of the Sardinian Hydrological Survey. The aim of this analysis is the application of downscaling procedures to space-time rainfall fields in case of orographic constraints.

The results have highlighted the existence of spatial heterogeneity in point rain gage measurements as well as in precipitation fields averaged on a regular spatial grid. This heterogeneity was partially considered by means of a locally defined modulating function which takes into account the different mean values of the precipitation intensity probability distributions depending on the altitude.

Due to the high space-time variability in the precipitation events, a great number of events has to be examined in order to retrieve local dependencies in rainfall distributions.
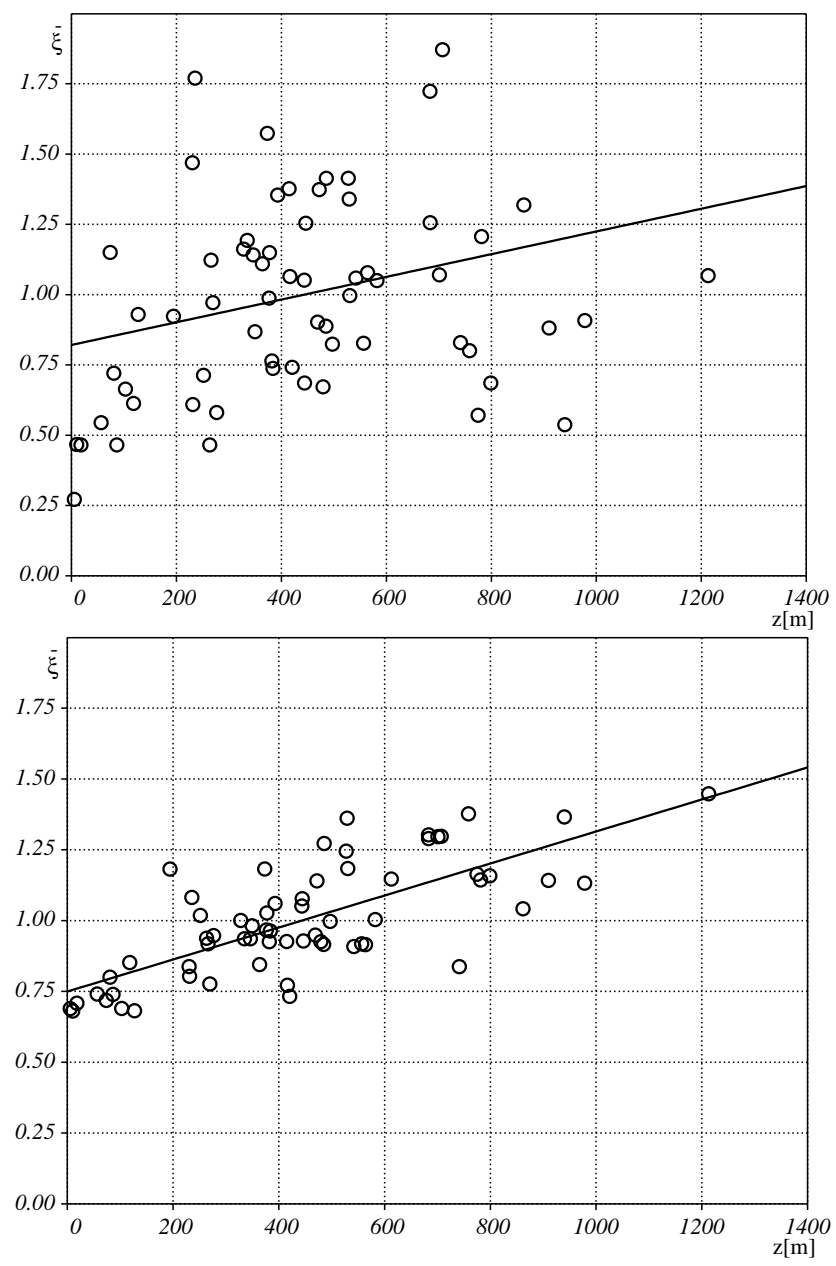

Fig. 10. Averages of the modulating function $\bar{\xi}$ plotted versus mean cell altitude $z$ for the events selected on the B domain. The plots refer to the observed sequences (top) and to one set of syntethic generations (bottom).

The dependency between modulating function and altitude was determined on precipitation data referring to more than 800 events. This relationship was used to make observed sequences spatially homogeneous as well as to introduce local orographic constraints in synthetic rainfall fields generated with a homogeneous and isotropic model.

The proposed downscaling procedure is based on the multifractal model STRAIN, with self-similar transformations between space and time scales. The model parsimoniousness allowed to keep only one free parameter depending on the mean rainfall intensity $I$, that should be predicted by meteorological models.

The orographic influences highlighted in the analysis are finally introduced multiplying synthetic fields obtained with the STRAIN model by a modulating function depending on the altitude of the grid cells. According to the obtained results the proposed methodology appears suitable in the generation of rainfall fields also in case of a mountainous terrain.

A final comment regards the way the multifractal analysis was here conducted. Indeed deriving rainfall fields from 
point measurements may affect the estimates of multifractal exponents and consequently the downscaling model calibration. It would be advisable to understand the effect induced by the use of point measures instead of areal ones. Unfortunately we were not able to perform in the analyzed region this kind of investigation due to the lack of areal measures . This research issue can be better investigated in orographic regions where both radar data and rain gage measurements are simultaneously available.

Acknowledgements. We are grateful to Sardinian Hydrological Survey for making data of the high temporal resolution rain gage network available. The research was supported by the Italian Ministry of Education, University and Research (MIUR) - Programma Operativo Nazionale - Misura 1.3, Prot. 13018/2001.

\section{Edited by: L. Ferraris}

Reviewed by: anonymous referees

\section{References}

Beven, K. J.: Rainfall-Runoff Modelling: The Primer, Wiley, Chichester, 2001.

Buizza, R., Miller, M., and Palmer, T. N.: Stochastic simulation of model uncertainty in the ECMWF ensemble prediction system, Quart. J. Roy. Meteor. Soc., 125, 2887-2908, 1999.

Deidda, R.: Rainfall downscaling in a space-time multifractal framework, Water Resour. Res., 36, 1779-1794, 2000.
Deidda, R., Badas, M. G., and Piga, E.: Space-time scaling in highintensity Tropical Ocean Global Atmosphere Coupled OceanAtmosphere Response Experiment TOGA-COARE storms, Water Resour. Res., 40, W02 506, doi:10.1029/2003WR002 574, 2004.

Deidda, R., Badas, M. G., and Piga, E.: Space-time multifractality of remotely sensed rainfall fields, J. Hydrol., in press (corrected proof available online), 29 April 2005.

Ferraris, L., Rudari, R., and Siccardi, F.: The uncertainty in the prediction of flash floods in the northern mediterranean environment, J. Hydrometeorology, 3, 714-727, 2002.

Harris, D., Menabde, M., Seed, A. W., and Austin, G.: Multiscaling characterization of rain fields with a strong orographic influence, J. Geophys. Res., 101(D21), 26 405-26414, 1996.

Jothityangkoon, C., Sivapalan, M., and Viney, N. R.: Tests of a space-time model of daily rainfall in southwest Australia based on nonhomogeneous random cascades, Water Resour. Res., 36, 267-284, 2000.

Lovejoy, S. and Schertzer, D.: Generalized scale invariance and fractal models of rain, Water Resour. Res., 21, 1233-1250, 1985.

Pathirana, A. and Herath, S.: Multifractal modelling and simulation of rain fields exhibiting spatial heterogeneity, Hydrol. Earth Syst. Sci., 6(4), 659-708, 2002.

Purdy, J. C., Harris, D., Austin, G. L., Seed, A. W., and Gray, W.: A case study of orographic rainfall processes incorporating multiscaling characterization techniques, J. Geophys. Res., 106(D8), 7837-7845, 2001. 